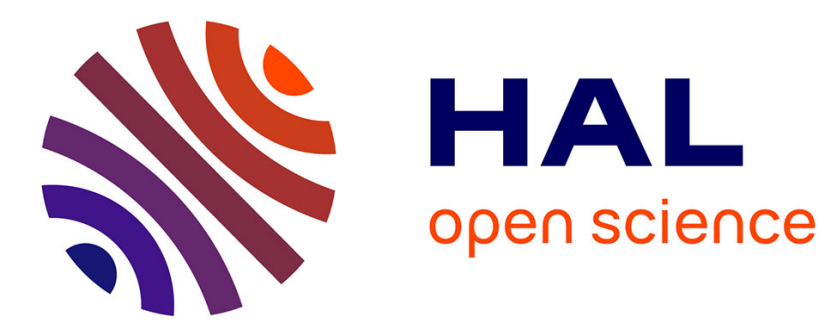

\title{
A quadratic Lyapunov function for Saint-Venant equations with arbitrary friction and space-varying slope
}

\author{
Amaury Hayat, Peipei Shang
}

\section{To cite this version:}

Amaury Hayat, Peipei Shang. A quadratic Lyapunov function for Saint-Venant equations with arbitrary friction and space-varying slope. Automatica, 2019, 100, pp.52-60. hal-01704710

\author{
HAL Id: hal-01704710 \\ https://hal.science/hal-01704710
}

Submitted on 8 Feb 2018

HAL is a multi-disciplinary open access archive for the deposit and dissemination of scientific research documents, whether they are published or not. The documents may come from teaching and research institutions in France or abroad, or from public or private research centers.
L'archive ouverte pluridisciplinaire HAL, est destinée au dépôt et à la diffusion de documents scientifiques de niveau recherche, publiés ou non, émanant des établissements d'enseignement et de recherche français ou étrangers, des laboratoires publics ou privés. 


\title{
A quadratic Lyapunov function for Saint-Venant equations with arbitrary friction and space-varying slope ${ }^{\star}$
}

\author{
Amaury Hayat ${ }^{a}$, Peipei Shang ${ }^{\text {a,b }}$, \\ a Sorbonne Universités, UPMC Univ. Paris 06, UMR 7598, Laboratoire Jacques-Louis Lions, 4 place Jussieu, F-75252 Paris, \\ France \\ ${ }^{\mathrm{b}}$ School of Mathematical Sciences, Tongji University, Shanghai 200092, China
}

\begin{abstract}
The exponential stability problem of the nonlinear Saint-Venant equations is addressed in this paper. We consider the general case where an arbitrary friction and space-varying slope are both included in the system, which lead to non-uniform steady-states. An explicit quadratic Lyapunov function as a weighted function of a small perturbation of the steady-states is constructed. Then we show that by a suitable choice of boundary feedback controls, that we give explicitly, the local exponential stability of the nonlinear Saint-Venant equations for the $H^{2}$-norm is guaranteed.
\end{abstract}

Key words: asymptotic stabilization; Lyapunov; Saint-Venant equations; inhomogeneous; robust control of nonlinear systems.

\section{Introduction}

Since discovered in 1871 by Barré de Saint-Venant [16], the shallow water equations (or Saint-Venant equations in unidimensional form) have been frequently used by hydraulic engineers in their practice. Their apparent simplicity and their ability to describe fairly well the behaviour of rivers and water channel make them a useful tool for many applications as for instance the regulation of navigable rivers and irrigation networks in agriculture. Among which, the problem of designing control tools to regularize the water level and the flow rate in the open hydraulic systems has been studied for a long time $[15,24,30-32]$.

The Saint-Venant equations constitute a nonlinear $2 \times 2$ 1-D hyperbolic system. In the last decades, the boundary feedback stabilization problem for 1-D hyperbolic systems has been widely investigated, and many tools have been developed. To our knowledge, the first result for nonlinear $2 \times 2$ homogeneous systems was obtained by Greenberg and $\mathrm{Li}$ [23] in the framework of $C^{1}$ solutions by using the characteristic method. Later on, this result was generalized by Qin [34] to $n \times n$ homoge-

\footnotetext{
* This paper was not presented at any IFAC meeting. Corresponding author Peipei Shang.

Email addresses: hayat@ljll.math.upmc.fr (Amaury Hayat), peipeishang@hotmail.com (Peipei Shang).
}

neous systems. In 1999, Coron et al. introduced another method: the quadratic Lyapunov function, firstly used to analyze the asymptotic behavior of linear hyperbolic equations in the $L^{2}$ norm but then generalized for nonlinear hyperbolic equations in the framework of $C^{1}$ and $H^{2}$ solutions [8-11]. Both of these two methods guarantee the exponential stability of the nonlinear homogeneous hyperbolic systems when the boundary conditions satisfy an appropriate sufficient dissipativity property. Such boundary conditions are the so-called static boundary feedback control and lead to feedbacks that only depend on the measures at the boundaries. However, when inhomogenous systems are considered, it is usually difficult (or even impossible) to construct a quadratic Lyapunov function with static boundary feedback [3, Chapter 5.6] or in [2]. The backstepping method introduced by Krstic et al. in [29] is a powerful tool to deal with the exponential stabilization of inhomogenous hyperbolic systems. Initially developed for parabolic equations [35], this method has been firstly applied to first order hyperbolic equations in [28], and then generalized to $n+1 \times n+1$ linear hyperbolic systems with $n$ positive and one negative characteristic speed in $[36,18]$. The case of general bidirectional linear systems was recently treated in [26]. For the nonlinear case, one can refer to [14], where the authors designed a full-state feedback control actuated on only one boundary and achieved exponential stability for the closed-loop $2 \times 2$ quasilinear hyperbolic systems in $H^{2}$-norm. Later on, this result was 
generalized in [27] to $n \times n$ quasilinear hyperbolic systems. In particular, in the context of the Saint-Venant equations, the backstepping method has been used to achieve exponential stabilization of the linearized SaintVenant Exner equations with arbitrary slope or friction in [20], both in subcritical and supercritical regime. It has also been used in [21] to stabilize a linearized bilayered Saint-Venant model, a $4 \times 4$ system of two SaintVenant systems interacting with each other. With the backstepping method, one can realize rapid decay (i.e., exponential decay with arbitrary rate) or even finite time stabilization for some linear case $[1,12,26]$. However, one requires a full-state feedback control rather than static boundary feedback control depending only on the values at the boundaries. Nevertheless, in some cases, it is possible to design an observer to tackle this issue $[17,19,20,36]$. In this paper, we will use a direct Lyapunov approach to study the exponential stability for nonhomogeneous Saint-Venant equations with arbitrary friction and space-varying slope. The advantage is that using this method, we only need to measure the value at the boundary, which is much easier for practical implementations.

The first result concerning this method applied to SaintVenant equations was obtained by Coron et al. in 1999 for the homogeneous case, i.e., without any friction or slope [10]. There, they use an entropy of the system as a Lyapunov function. But this Lyapunov function has only a semi-negative definite time derivative. One has to conclude the stability result using LaSalle's invariance principle which is usually difficult to apply due to the problem of precompactness of the trajectories. Later on, the authors introduce in [11] a strict Lyapunov function for conservation laws that can be diagonalized with Riemann invariants. The time derivative of the Lyapunov function can be made strictly negative definite by an appropriate choice of the boundary conditions. They apply the result to regulate the level and flow in an open channel without friction or slope. Under the assumption that the friction and the slope are sufficiently small in $C^{1}$-norm, the stability may be proved using the method of characteristics as in $[22,33]$ or using a Lyapunov approach as in $[7,9]$. In the special case where the slope is a constant and "compensate" with the influence of the friction, thus resulting the uniform steady-states, the stability of the linearized system was considered in [5]. There, the authors use the same Lyapunov function as in [11]. More recently, in [4], the authors managed to study the stability in $H^{2}$-norm of the nonlinear and nonhomogeneous system when the friction is arbitrary but in the absence of the slope. It should be noted that this last result was proved using a basic quadratic Lyapunov function (see [2] for a proper definition) independent of the length of the water channel which is not trivial as, usually, the existence of a basic quadratic Lyapunov function for a nonhomogenous system depends on the size of the domain [25].
Of course for realistic description of the behaviour of rivers one can easily understand that adding a slope is essential not only because it is the prime mover of the flow but also because in some common cases the effect of the slope can be much larger than the effect of the friction, both being non-negligible: it is the steep-slope regime (see for instance [6, Chapter 5-3]). To our knowledge, no study so far takes into account a non-negligible slope except in the special case mentioned previously where the slope compensate exactly the friction and cancels the source term [5].

Our contribution in this paper is that we managed to construct an explicit Lyapunov control function to analyze the local exponential stability in $H^{2}$-norm of the nonlinear Saint-Venant equations in the case where both the friction and the slope are taken into account and are arbitrary (not necessarily small). Especially we deal with the case where the slope may vary with respect to the space variable. This is all the more important that the slope is likely to vary in a river, even sometimes on short distances. We first describe three regimes depending whether the influence of the slope is smaller, equal or greater to the influence of the friction and we show that the dynamics in two opposite regimes are inverted. Then we construct a quadratic Lyapunov function for the $H^{2}$-norm whatever the friction and the slope are.

The organization of the paper is as follows. In Section 2 , we give a description of the non-linear Saint-Venant equations together with some definitions and we state our main result (Theorem 3). In Section 3, the exponential stability of the linearized system is firstly studied by constructing a quadratic Lyapunov function. Based on the results of the linearized system, we then show that a similar expanded Lyapunov function enables us to get the exponential stability of the nonlinear system by properly choosing the boundary feedback controls.

\section{Description of the Saint-Venant equations and the main result}

The non-linear Saint-Venant equations with slope and friction are given by the following system:

$$
\begin{gathered}
\partial_{t} H+\partial_{x}(H V)=0 \\
\partial_{t} V+\partial_{x}\left(\frac{V^{2}}{2}+g H\right)+\left(\frac{k V^{2}}{H}-g C\right)=0
\end{gathered}
$$

where $H(t, x)$ is the water depth, $V(t, x)$ is the horizontal water velocity. The slope $C(\cdot) \in C^{2}([0, L])$ is defined by $C(x)=-\frac{d B}{d x}$ with $B(x)$ the elevation of the bottom, $g$ is the constant gravity acceleration and $k$ is a constant friction coefficient. Note from (1) and (2) that the equi- 
librium $H^{*}$ and $V^{*}$ verifies:

$$
\begin{gathered}
H^{*}(x) V^{*}(x)=Q^{*} \\
V^{*} V_{x}^{*}+g H_{x}^{*}+\left(\frac{k V^{* 2}}{H^{*}}-g C\right)=0 .
\end{gathered}
$$

As we are interested in physical stationary states, we suppose that $H^{*}>0$ and $V^{*}>0$. Therefore $Q^{*}>0$ is any given constant set point and corresponds to the flow rate. Substituting (3) to (4), we get that $V^{*}$ satisfies

$$
V_{x}^{*}=\frac{V^{* 2}\left(\frac{k V^{* 3}}{Q^{*}}-g C\right)}{g Q^{*}-V^{* 3}} .
$$

Observe that the steady-states are therefore non necessary uniform. As we are interested in navigable rivers we also suppose that the flow is in the fluvial regime, i.e.,

$$
g H^{*}>V^{* 2}
$$

or equivalently $g Q^{*}-V^{* 3}>0$. Then the system (1) and (2) has a positive and a negative eigenvalue and for any flow rate $Q^{*}$, equation (5) has a unique $C^{1}$ and even $C^{\infty}$ solution on $[0, L]$ with any given boundary data $V^{*}(0)=V_{0}^{*}$. Moreover, the steady-states have three possible dynamics depending on the slope as the following.

(1) When $g C<\frac{k V^{* 2}}{H^{*}}$, also known in hydraulic engineering as "mild slope regime". This covers also the case without slope. Note from (3) and (5) that in this case, $H^{*}$ decreases while $V^{*}$ increases and consequently the system becomes closer to the critical point where $g H^{*}=V^{* 2}$ which is the limit of the fluvial regime.

(2) When $g C=\frac{k V^{* 2}}{H^{*}}$, which means that the friction and the slope "compensate" each other. When the slope $C$ is in additionally constant, the steadystates are uniform. This special case has been studied in [5] and only for the linearized system.

(3) When $g C>\frac{k V^{* 2}}{H^{*}}$, also known in hydraulic engineering as "steep slope regime". Then the dynamics of the steady-states are inverted: $H^{*}$ tends to increase while $V^{*}$ decreases and consequently the system moves away from the limit of the fluvial regime defined by the critical point where $g H^{*}=V^{* 2}$.

Our goal is to ensure the exponential stability of the steady-states of the nonlinear system (1) and (2) for all the above three cases under some boundary conditions of the form:

$$
V(t, 0)=\mathcal{B}(H(t, 0)), \quad V(t, L)=\mathcal{B}(H(t, L)),
$$

where $\mathcal{B}: \mathbb{R} \rightarrow \mathbb{R}$ is of class $C^{2}$. These kind of boundary conditions are imposed by physical devices located at the ends of the channel where the controls are implemented, as for instance mobile spillways or tunable hydraulic gates as in irrigation canals and navigable rivers. For these two examples, some more detail and explicit expressions of the boundary conditions are given in [3].

We will first prove the exponential stability for the linearized system for the $L^{2}$-norm. Note that for nonlinear systems, the stability depends on the topology considered as shown in [13]. In this paper, we will consider the exponential stability in $H^{2}$-norm.

For any given initial condition

$$
H(0, x)=H_{0}(x), \quad V(0, x)=V_{0}(x), \quad x \in[0, L],
$$

we suppose that the following compatibility conditions hold

$$
\begin{aligned}
& V_{0}(0)=\mathcal{B}\left(H_{0}(0)\right), \quad V_{0}(L)=\mathcal{B}\left(H_{0}(L)\right), \\
& \partial_{x}\left(\frac{V_{0}^{2}}{2}+g H_{0}\right)(0)+\frac{k V_{0}^{2}}{H_{0}}(0)-g C(0) \\
& =\mathcal{B}^{\prime}\left(H_{0}(0)\right) \partial_{x}\left(H_{0} V_{0}\right)(0), \\
& \partial_{x}\left(\frac{V_{0}^{2}}{2}+g H_{0}\right)(L)+\frac{k V_{0}^{2}}{H_{0}}(L)-g C(L) \\
& =\mathcal{B}^{\prime}\left(H_{0}(L)\right) \partial_{x}\left(H_{0} V_{0}\right)(L) .
\end{aligned}
$$

These compatibility conditions guarantee the wellposedness of the system (1), (2), (7) and (8) for sufficiently small initial data. More precisely, we have (see $[3$, Appendix B])

Theorem 1 There exists $\delta_{0}>0$ such that for every $\left(H_{0}, V_{0}\right)^{T} \in H^{2}\left((0, L) ; \mathbb{R}^{2}\right)$ satisfying

$$
\left\|\left(H_{0}-H^{*}, V_{0}-V^{*}\right)^{T}\right\|_{H^{2}\left((0, L) ; \mathbb{R}^{2}\right)} \leq \delta_{0}
$$

and compatibility conditions (9) to (11). The Cauchy problem (1), (2), (7) and (8) has a unique maximal classical solution

$$
(H, V)^{T} \in C^{0}\left([0, T) ; H^{2}\left((0, L) ; \mathbb{R}^{2}\right)\right)
$$

with $T \in(0,+\infty)$.

We recall the definition of the exponential stability in $H^{2}$-norm:

Definition 2 The steady-state $\left(H^{*}, V^{*}\right)^{T}$ of the system (1), (2) and (7) is exponentially stable for the $\mathrm{H}^{2}$-norm if there exist $\gamma>0, \delta>0$ and $C>0$ such that for every $\left(H_{0}, V_{0}\right)^{T} \in H^{2}\left((0, L) ; \mathbb{R}^{2}\right)$ satisfying $\|\left(H_{0}-H^{*}, V_{0}-\right.$ $\left.V^{*}\right)^{T} \|_{H^{2}\left((0, L) ; \mathbb{R}^{2}\right)} \leq \delta$ and the compatibility conditions (9) to (11), the Cauchy problem (1), (2), (7) and (8) has a 
unique classical solution on $[0,+\infty) \times[0, L]$ and satisfies

$$
\begin{aligned}
& \left\|\left(H(t, \cdot)-H^{*}, V(t, \cdot)-V^{*}\right)^{T}\right\|_{H^{2}\left((0, L) ; \mathbb{R}^{2}\right)} \\
\leq & C e^{-\gamma t}\left\|\left(H_{0}-H^{*}, V_{0}-V^{*}\right)^{T}\right\|_{H^{2}\left((0, L) ; \mathbb{R}^{2}\right)},
\end{aligned}
$$

for all $t \in[0,+\infty)$.

Based on this definition, our main result is the following:

Theorem 3 The nonlinear Saint-Venant system (1), (2) and (7) is exponentially stable for the $\mathrm{H}^{2}{ }_{-}$norm provided that the boundary conditions satisfy

$$
\begin{aligned}
\mathcal{B}^{\prime}\left(H^{*}(0)\right) & \in\left(-\frac{g}{V^{*}(0)},-\frac{V^{*}(0)}{H^{*}(0)}\right), \\
\text { and } \quad \mathcal{B}^{\prime}\left(H^{*}(L)\right) & \in \mathbb{R} \backslash\left[-\frac{g}{V^{*}(L)},-\frac{V^{*}(L)}{H^{*}(L)}\right] .
\end{aligned}
$$

The proof of this theorem is given in Section 3. To that end, we will first prove the exponential stability result (Proposition 4) for the linearized system for the $L^{2}$-norm by finding a suitable Lyapunov function. Then we show that this Lyapunov function enables us to obtain the exponential stability for the $H^{2}$-norm for the nonlinear system under boundary control conditions (7) with properties (14).

\section{Exponential stability for the $H^{2}$-norm with ar- bitrary friction and space-varing slope}

\subsection{Exponential stability of the linearized system}

In this section, we study the exponential stability of the linearized system about a steady-state $\left(H^{*}, V^{*}\right)^{T}$ for the $L^{2}$-norm. We define the perturbation functions $h$ and $v$ as

$$
\begin{aligned}
h(t, x) & =H(t, x)-H^{*}(x), \\
v(t, x) & =V(t, x)-V^{*}(x) .
\end{aligned}
$$

The linearization of the system (1) and (2) about the steady-state is

$$
\left(\begin{array}{l}
h \\
v
\end{array}\right)_{t}+\left(\begin{array}{cc}
V^{*} & H^{*} \\
g & V^{*}
\end{array}\right)\left(\begin{array}{l}
h \\
v
\end{array}\right)_{x}+\left(\begin{array}{cc}
V_{x}^{*} & H_{x}^{*} \\
f_{H}^{*} & V_{x}^{*}+f_{V}^{*}
\end{array}\right)\left(\begin{array}{l}
h \\
v
\end{array}\right)=0
$$

where $f_{H}^{*}$ and $f_{V}^{*}$ are defined by

$$
\begin{aligned}
f_{V}^{*} & =\frac{2 k V^{*}}{H^{*}}, \\
f_{H}^{*} & =-\frac{k V^{* 2}}{H^{* 2}} .
\end{aligned}
$$

The corresponding linearization of the boundary conditions (7) are given by

$$
\begin{gathered}
v(t, 0)=b_{0} h(t, 0), \\
v(t, L)=b_{1} h(t, L),
\end{gathered}
$$

where

$$
b_{0}=\mathcal{B}^{\prime}\left(H^{*}(0)\right), \quad b_{1}=\mathcal{B}^{\prime}\left(H^{*}(L)\right) .
$$

The initial condition is given as follows

$$
h(0, x)=h_{0}(x), \quad v(0, x)=v_{0}(x),
$$

where $\left(h_{0}, v_{0}\right)^{T} \in L^{2}\left((0, L) ; \mathbb{R}^{2}\right)$. The Cauchy problem (17), (20) and (22) is well-posed (see [3, Appendix A]). Note that the exponential stability of the linearized system is now a problem of null-stabilization for $h$ and $v$. We have the following result:

Proposition 4 For the linearized Saint-Venant system (17), (20) and (22), if the boundary conditions satisfy

$b_{0} \in\left(-\frac{g}{V^{*}(0)},-\frac{V^{*}(0)}{H^{*}(0)}\right), b_{1} \in \mathbb{R} \backslash\left[-\frac{g}{V^{*}(L)},-\frac{V^{*}(L)}{H^{*}(L)}\right]$.

Then there exists a constant $\mu>0, q_{1} \in C^{1}([0, L] ;(0,+\infty))$, $q_{2} \in C^{1}([0, L] ;(0,+\infty))$ and $\delta>0$ such that the following control Lyapunov function candidate

$V(h, v)=\int_{0}^{L} \frac{q_{1}+q_{2}}{H^{*}}\left(g h^{2}+2 \frac{q_{1}-q_{2}}{q_{1}+q_{2}} \sqrt{g H^{*}} h v+H^{*} v^{2}\right) d x$

verifies:

$$
V(h, v) \geq \delta\left(\|h\|_{L^{2}(0, L)}^{2}+\|v\|_{L^{2}(0, L)}^{2}\right)
$$

for any $(h, v) \in L^{2}\left((0, L) ; \mathbb{R}^{2}\right)$. If in addition, $(h, v)^{T}$ is a solution of the system (17), (20) and (22), we have

$$
\frac{d}{d t}(V(h(t, \cdot), v(t, \cdot))) \leq-\mu V(h(t, \cdot), v(t, \cdot))
$$

in the distribution sense which implies the exponential stability of the linearized system (17), (20) and (22) for the $L^{2}$-norm.

In order to prove Proposition 4, we introduce the following lemma, the proof of which is given in the Appendix.

Lemma 5 The function $\eta$ defined by

$$
\eta=\frac{\lambda_{2}}{\lambda_{1}} \varphi
$$

is a solution to the equation

$$
\eta^{\prime}=\left|\frac{a}{\lambda_{1}}+\frac{b}{\lambda_{2}} \eta^{2}\right|
$$


where $\lambda_{1}$ and $\lambda_{2}$ are defined in (29), $\varphi$ is given by (38), $a$ and $b$ are given by (41) below.

PROOF. [Proof of Proposition 4] Let us denote

$$
A(x)=\left(\begin{array}{cc}
V^{*} & H^{*} \\
g & V^{*}
\end{array}\right)
$$

Under the subcritical condition (6), the matrix $A(x)$ has two real distinct eigenvalues $\lambda_{1}$ and $-\lambda_{2}$ with

$$
\lambda_{1}(x)=\sqrt{g H^{*}}+V^{*}>0, \lambda_{2}(x)=\sqrt{g H^{*}}-V^{*}>0 .
$$

We define the characteristic coordinates as follows

$$
\left(\begin{array}{l}
\xi_{1} \\
\xi_{2}
\end{array}\right)=\left(\begin{array}{cc}
\sqrt{\frac{g}{H^{*}}} & 1 \\
-\sqrt{\frac{g}{H^{*}}} & 1
\end{array}\right)\left(\begin{array}{l}
h \\
v
\end{array}\right)
$$

With these definitions and notations, the linearized Saint-Venant equations (17) are written in characteristic form:

$$
\left(\begin{array}{l}
\xi_{1} \\
\xi_{2}
\end{array}\right)_{t}+\left(\begin{array}{cc}
\lambda_{1} & 0 \\
0 & -\lambda_{2}
\end{array}\right)\left(\begin{array}{l}
\xi_{1} \\
\xi_{2}
\end{array}\right)_{x}+\left(\begin{array}{ll}
\gamma_{1} & \delta_{1} \\
\gamma_{2} & \delta_{2}
\end{array}\right)\left(\begin{array}{l}
\xi_{1} \\
\xi_{2}
\end{array}\right)=0
$$

In $(31)$,

$$
\begin{aligned}
& \gamma_{1}(x)=-\frac{3 f\left(H^{*}, V^{*}\right)}{4\left(\sqrt{g H^{*}}+V^{*}\right)}+\frac{k V^{*}}{H^{*}}-\frac{k V^{* 2}}{2 H^{*} \sqrt{g H^{*}}}, \\
& \delta_{1}(x)=-\frac{f\left(H^{*}, V^{*}\right)}{4\left(\sqrt{g H^{*}}+V^{*}\right)}+\frac{k V^{*}}{H^{*}}+\frac{k V^{* 2}}{2 H^{*} \sqrt{g H^{*}}}, \\
& \gamma_{2}(x)=\frac{f\left(H^{*}, V^{*}\right)}{4\left(\sqrt{g H^{*}}-V^{*}\right)}+\frac{k V^{*}}{H^{*}}-\frac{k V^{* 2}}{2 H^{*} \sqrt{g H^{*}}}, \\
& \delta_{2}(x)=\frac{3 f\left(H^{*}, V^{*}\right)}{4\left(\sqrt{g H^{*}}-V^{*}\right)}+\frac{k V^{*}}{H^{*}}+\frac{k V^{* 2}}{2 H^{*} \sqrt{g H^{*}}},
\end{aligned}
$$

where $f\left(H^{*}, V^{*}\right)=\frac{k V^{* 2}}{H^{*}}-g C$.

As the diagonal coefficients of the source term in (31) may bring complexity on the analysis of the stability, we then make a coordinate transformation inspired by [29] (see also [4]) to remove the diagonal coefficients. We introduce the notations

$$
\begin{aligned}
\varphi_{1}(x) & =\exp \left(\int_{0}^{x} \frac{\gamma_{1}(s)}{\lambda_{1}(s)} d s\right), \\
\varphi_{2}(x) & =\exp \left(-\int_{0}^{x} \frac{\delta_{2}(s)}{\lambda_{2}(s)} d s\right), \\
\varphi(x) & =\frac{\varphi_{1}(x)}{\varphi_{2}(x)}
\end{aligned}
$$

and the new coordinates

$$
\left(\begin{array}{l}
y_{1} \\
y_{2}
\end{array}\right)=\left(\begin{array}{cc}
\varphi_{1} & 0 \\
0 & \varphi_{2}
\end{array}\right)\left(\begin{array}{l}
\xi_{1} \\
\xi_{2}
\end{array}\right)
$$

Then system (31) is transformed into the following system expressed in the new coordinates

$$
\left(\begin{array}{l}
y_{1} \\
y_{2}
\end{array}\right)_{t}+\left(\begin{array}{cc}
\lambda_{1} & 0 \\
0 & -\lambda_{2}
\end{array}\right)\left(\begin{array}{l}
y_{1} \\
y_{2}
\end{array}\right)_{x}+\left(\begin{array}{cc}
0 & a(x) \\
b(x) & 0
\end{array}\right)\left(\begin{array}{l}
y_{1} \\
y_{2}
\end{array}\right)=0
$$

with

$$
a(x)=\varphi(x) \delta_{1}(x), \quad b(x)=\varphi^{-1}(x) \gamma_{2}(x) .
$$

From (20), (30) and (39), we obtain the following boundary conditions for system (40)

$$
\begin{aligned}
y_{1}(t, 0) & =k_{0} \frac{\varphi_{1}(0)}{\varphi_{2}(0)} y_{2}(t, 0), \\
y_{2}(t, L) & =k_{1} \frac{\varphi_{2}(L)}{\varphi_{1}(L)} y_{1}(t, L),
\end{aligned}
$$

where

$k_{0}=\frac{b_{0} H^{*}(0)+\sqrt{g H^{*}(0)}}{b_{0} H^{*}(0)-\sqrt{g H^{*}(0)}}, k_{1}=\frac{b_{1} H^{*}(L)-\sqrt{g H^{*}(L)}}{b_{1} H^{*}(L)+\sqrt{g H^{*}(L)}}$.

Note that from (43), it is easy to check that condition (23), using our notation (29), is equivalent to

$$
k_{0}^{2}<\left(\frac{\lambda_{2}(0)}{\lambda_{1}(0)}\right)^{2}, \quad k_{1}^{2}<\left(\frac{\lambda_{1}(L)}{\lambda_{2}(L)}\right)^{2} .
$$

Let us define

$V: \quad L^{2}(0, L) \times L^{2}(0, L) \rightarrow \mathbb{R}^{+}$

$V\left(\psi_{1}, \psi_{2}\right)=\int_{0}^{L}\left(f_{1}(x) \psi_{1}^{2}(x) e^{-\frac{\mu}{\lambda_{1}} x}+f_{2}(x) \psi_{2}^{2}(x) e^{\frac{\mu}{\lambda_{2}} x}\right) d x$

where the parameter $\mu>0$ and two functions $f_{1} \in$ $C^{1}([0, L] ;(0,+\infty))$ and $f_{2} \in C^{1}([0, L] ;(0,+\infty))$ are to be determined. Obviously, there exists $\delta>0$ such that for any $\left(\psi_{1}, \psi_{2}\right) \in L^{2}\left((0, L) ; \mathbb{R}^{2}\right)$

$$
V\left(\psi_{1}, \psi_{2}\right) \geq \bar{\delta}\left(\left\|\psi_{1}\right\|_{L^{2}(0, L)}^{2}+\left\|\psi_{2}\right\|_{L^{2}(0, L)}^{2}\right) .
$$

For any arbitrary $C^{1}$-solution $y_{1}$ and $y_{2}$ to system (40) and (42), we denote $V(t)$ by

$$
V(t)=V\left(y_{1}(t, \cdot), y_{2}(t, \cdot)\right)
$$


From (45) and differentiating $V$ with respect to time $t$ we get

$$
\begin{aligned}
\frac{d V}{d t}= & -\mu V-\left[\lambda_{1} f_{1} e^{-\frac{\mu}{\lambda_{1}} x} y_{1}^{2}-\lambda_{2} f_{2} e^{\frac{\mu}{\lambda_{2}} x} y_{2}^{2}\right]_{0}^{L} \\
& -\int_{0}^{L}\left[-\left(\lambda_{1} f_{1}\right)_{x} e^{-\frac{\mu}{\lambda_{1}} x} y_{1}^{2}+\left(\lambda_{2} f_{2}\right)_{x} e^{\frac{\mu}{\lambda_{2}} x} y_{2}^{2}\right. \\
& \left.+2\left(f_{1} e^{-\frac{\mu}{\lambda_{1}} x} a(x)+f_{2} e^{\frac{\mu}{\lambda_{2}} x} b(x)\right) y_{1} y_{2}\right] d x
\end{aligned}
$$

We observe that in (48), there is a term relying on the boundary controls that will be chosen to make this term negative along the system trajectories. Moreover, there also appears to have an interior term which is intrinsic to the system. Let us deal firstly with the interior term, we denote by

$$
\begin{aligned}
I_{1}:=\int_{0}^{L} & {\left[-\left(\lambda_{1} f_{1}\right)_{x} e^{-\frac{\mu}{\lambda_{1}} x} y_{1}^{2}+\left(\lambda_{2} f_{2}\right)_{x} e^{\frac{\mu}{\lambda_{2}} x} y_{2}^{2}\right.} \\
& \left.+2\left(f_{1} e^{-\frac{\mu}{\lambda_{1}} x} a(x)+f_{2} e^{\frac{\mu}{\lambda_{2}} x} b(x)\right) y_{1} y_{2}\right] d x
\end{aligned}
$$

To ensure that there exists $\mu_{1}>0$ such that for all $\mu \in\left(0, \mu_{1}\right], I_{1}$ is positive for any $t>0$ and any solution $\left(y_{1}, y_{2}\right)$, one only needs to construct $f_{1}$ and $f_{2}$ to guarantee that for any $x \in[0, L]$

$$
\begin{gathered}
-\left(\lambda_{1} f_{1}\right)_{x}>0, \quad\left(\lambda_{2} f_{2}\right)_{x}>0 \\
-\left(\lambda_{1} f_{1}\right)_{x}\left(\lambda_{2} f_{2}\right)_{x}-\left(f_{1}(x) a(x)+f_{2}(x) b(x)\right)^{2}>0 .
\end{gathered}
$$

Indeed in this case from the strict inequality in (50) and (51), there exists $\mu_{1}>0$ such that for all $\mu \in\left(0, \mu_{1}\right]$,

$$
\begin{gathered}
-\left(\lambda_{1} f_{1}\right)_{x} e^{-\frac{\mu}{\lambda_{1}} x}>0, \quad\left(\lambda_{2} f_{2}\right)_{x} e^{\frac{\mu}{\lambda_{2}} x}>0, \\
-\left(\lambda_{1} f_{1}\right)_{x} e^{-\frac{\mu}{\lambda_{1}} x}\left(\lambda_{2} f_{2}\right)_{x} e^{\frac{\mu}{\lambda_{2}} x} \\
-\left(f_{1}(x) e^{-\frac{\mu}{\lambda_{1}} x} a(x)+f_{2}(x) e^{\frac{\mu}{\lambda_{2}} x} b(x)\right)^{2}>0 .
\end{gathered}
$$

Let us point out that there exist $f_{1}$ and $f_{2}$ such that (50) and (51) hold as soon as there exists a positive function $\eta$ well defined on $[0, L]$ and satisfying the following equation (see [2])

$$
\eta^{\prime}=\left|\frac{a}{\lambda_{1}}+\frac{b}{\lambda_{2}} \eta^{2}\right|
$$

Therefore, one of the key points to prove Proposition 4 is to find a positive solution to (54). And from Lemma 5 we know that such solution does exist. Hence, we can define a map

$$
f:(\eta, \varepsilon) \rightarrow\left|\frac{a}{\lambda_{1}}+\frac{b}{\lambda_{2}} \eta^{2}\right|+\varepsilon,
$$

which is locally Lipschitz (and even $C^{1}$ ) in $\varepsilon$ around 0 . From Lemma 5 we know that

$$
\left\{\begin{array}{l}
\eta^{\prime}=f(\eta, 0) \\
\eta(0)=\frac{\lambda_{2}(0)}{\lambda_{1}(0)} \varphi(0)
\end{array}\right.
$$

admits a unique solution on $[0, L]$ which is given by $(27)$. Therefore, there exists $\varepsilon_{0}>0$ such that for all $\varepsilon \in\left[0, \varepsilon_{0}\right]$, the Cauchy problem

$$
\left\{\begin{array}{l}
\eta_{\varepsilon}^{\prime}=\left|\frac{a}{\lambda_{1}}+\frac{b}{\lambda_{2}} \eta_{\varepsilon}^{2}\right|+\varepsilon, \\
\eta_{\varepsilon}(0)=\frac{\lambda_{2}(0)}{\lambda_{1}(0)} \varphi(0)
\end{array}\right.
$$

admits a unique solution $\eta_{\varepsilon}$ on $[0, L]$. Moreover as $\eta_{\varepsilon}(0)>0$, we have $\eta_{\varepsilon}(x)>0$ for all $x \in[0, L]$. Now proceeding as in [2], we choose $f_{1}$ and $f_{2}$ as

$$
f_{1}=f_{1, \varepsilon}:=\frac{1}{\lambda_{1} \eta_{\varepsilon}}, \quad f_{2}=f_{2, \varepsilon}:=\frac{\eta_{\varepsilon}}{\lambda_{2}},
$$

then we have for any $\varepsilon \in\left(0, \varepsilon_{0}\right]$ that

$$
\begin{aligned}
-\left(\lambda_{1} f_{1, \varepsilon}\right)_{x}>0, & \left(\lambda_{2} f_{2, \varepsilon}\right)_{x}>0, \\
-\left(\lambda_{1} f_{1, \varepsilon}\right)_{x}\left(\lambda_{2} f_{2, \varepsilon}\right)_{x}- & \left(f_{1, \varepsilon} a+f_{2, \varepsilon} b\right)^{2} \\
= & \frac{\varepsilon^{2}+2 \varepsilon\left|\frac{a}{\lambda_{1}}+\frac{b}{\lambda_{2}} \eta_{\varepsilon}^{2}\right|}{\eta_{\varepsilon}^{2}}>0 .
\end{aligned}
$$

Thus, from (50), (51) and noticing the definition of $f_{1}$ and $f_{2}$ in (57), there exists $\mu_{1}>0$ such that for all $\mu \in\left(0, \mu_{1}\right], I_{1}$ defined by $(49)$ is positive for all $t \geq 0$.

Now, let us consider the boundary term in (48), we denote by

$$
I_{2}:=-\left[\lambda_{1} f_{1} e^{-\frac{\mu}{\lambda_{1}} x} y_{1}^{2}-\lambda_{2} f_{2} e^{\frac{\mu}{\lambda_{2}} x} y_{2}^{2}\right]_{0}^{L} .
$$

Suppose that (23) is satisfied, from (27), (44), (56) and (57), we have

$$
\begin{aligned}
& k_{0}^{2}<\left(\frac{\lambda_{2}(0)}{\lambda_{1}(0)}\right)^{2}=\frac{\lambda_{2}(0) f_{2,0}(0)}{\lambda_{1}(0) f_{1,0}(0)} \varphi^{-2}(0)=\frac{\lambda_{2}(0) f_{2, \varepsilon}(0)}{\lambda_{1}(0) f_{1, \varepsilon}(0)} \varphi^{-2}(0), \\
& k_{1}^{2}<\left(\frac{\lambda_{1}(L)}{\lambda_{2}(L)}\right)^{2}=\frac{\lambda_{1}(L) f_{1,0}(L)}{\lambda_{2}(L) f_{2,0}(L)} \varphi^{2}(L) .
\end{aligned}
$$

By the continuity of $f_{1, \varepsilon}(L)$ and $f_{2, \varepsilon}(L)$ with $\varepsilon$, there exists $0<\varepsilon_{1}<\varepsilon_{0}$ such that for any $\varepsilon \in\left(0, \varepsilon_{1}\right]$

$$
k_{1}^{2}<\frac{\lambda_{1}(L) f_{1, \varepsilon}(L)}{\lambda_{2}(L) f_{2, \varepsilon}(L)} \varphi^{2}(L),
$$


thus, there exists $0<\mu_{2}<\mu_{1}$ such that for any $\mu \in$ $\left(0, \mu_{2}\right]$

$$
k_{1}^{2}<\frac{\lambda_{1}(L) f_{1, \varepsilon}(L) e^{-\frac{\mu}{\lambda_{1}} L}}{\lambda_{2}(L) f_{2, \varepsilon}(L) e^{\frac{\mu}{\lambda_{2}} L} \varphi^{2}(L) .}
$$

Combining (61) and (64), we get

$$
\begin{aligned}
I_{2}= & -\left[\lambda_{1} f_{1} e^{-\frac{\mu}{\lambda_{1}} x} y_{1}^{2}-\lambda_{2} f_{2} e^{\frac{\mu}{\lambda_{2}} x} y_{2}^{2}\right]_{0}^{L} \\
= & \left(k_{1}^{2} \lambda_{2} f_{2, \varepsilon}(L) \varphi^{-2}(L) e^{\frac{\mu}{\lambda_{2}} L}-\lambda_{1} f_{1, \varepsilon}(L) e^{-\frac{\mu}{\lambda_{1}} L}\right) y_{1}^{2}(t, L) \\
& +\left(k_{0}^{2} \lambda_{1} f_{1, \varepsilon}(0) \varphi^{2}(0)-\lambda_{2} f_{2, \varepsilon}(0)\right) y_{2}^{2}(t, 0)<0 .
\end{aligned}
$$

From (48), (58), (59) and (65), we obtain

$$
\frac{d V}{d t}<-\mu V
$$

along the $C^{1}$-solutions of the system (40) and (42) for any $\mu \in\left(0, \mu_{2}\right]$. Since the $C^{1}$-solutions are dense in the set of $L^{2}$-solutions, inequality (66) also holds in the sense of distributions for the $L^{2}$-solutions (see [3, Section 2.1] for the details).

Let us define

$$
q_{1}:=f_{1} \varphi_{1}^{2} e^{-\frac{\mu}{\lambda_{1}} x} \quad \text { and } \quad q_{2}:=f_{2} \varphi_{2}^{2} e^{\frac{\mu}{\lambda_{2}} x} .
$$

For any $(h, v) \in L^{2}\left((0, L) ; \mathbb{R}^{2}\right)$, let $\left(\psi_{1}, \psi_{2}\right)$ be the result of the change of variable as in (30) and (39), we get immediately from (45) and (67) the expression of Lyapunov function candidate as in (24). Moreover, from (46), we have (25). From (66), we get (26) as well. The proof of Proposition 4 is completed.

Remark 6 Although the functions $f_{1}$ and $f_{2}$ defined in (57) are implicit, we can nevertheless construct explicit functions satisfying (50) and (51) based on the solution $\eta$ to (54) we have found, thus to get an explicit Lyapunov function. To be more precise, we consider the following two cases respectively:

(1) For the "mild slope regime" case, i.e., $g C<\frac{k V^{* 2}}{H^{*}}$, let

$$
f_{1}=\frac{1}{\lambda_{1}(\eta-\varepsilon)}, \quad f_{2}=\frac{\eta-\varepsilon}{\lambda_{2}},
$$

where we recall that $\eta=\frac{\lambda_{2}}{\lambda_{1}} \varphi$ is a solution to (54). It is easy to check that for $\varepsilon>0$ small enough, we have

$$
\begin{gathered}
-\left(\lambda_{1} f_{1}\right)_{x}>0, \quad\left(\lambda_{2} f_{2}\right)_{x}>0, \\
\text { and } \quad-\left(\lambda_{1} f_{1}\right)_{x}\left(\lambda_{2} f_{2}\right)_{x}>\left(f_{1} a+f_{2} b\right)^{2} .
\end{gathered}
$$

Noticing the definition of $\eta$ in (27), the negativeness of the boundary term (60) can be guaranteed by choosing \& small enough.
(2) For the "steep slope regime" case, i.e., $g C>\frac{k V^{* 2}}{H^{*}}$, let

$$
f_{1}=\frac{A}{\lambda_{1} \eta}, \quad f_{2}=\frac{B \eta}{\lambda_{2}},
$$

where $A$ and $B$ are two positive constants to be determined. Noticing (54), we have

$$
-\left(\lambda_{1} f_{1}\right)_{x}=\frac{A \eta^{\prime}}{\eta^{2}}>0, \quad\left(\lambda_{2} f_{2}\right)_{x}=B \eta^{\prime}>0
$$

and

$$
-\left(\lambda_{1} f_{1}\right)_{x}\left(\lambda_{2} f_{2}\right)_{x}=\frac{A B\left(\eta^{\prime}\right)^{2}}{\eta^{2}}=\frac{A B\left(\frac{a}{\lambda_{1}}+\frac{b}{\lambda_{2}} \eta^{2}\right)^{2}}{\eta^{2}} .
$$

Moreover, we have

$\left(f_{1} a+f_{2} b\right)^{2}=\left(\frac{A a}{\lambda_{1} \eta}+\frac{b B \eta}{\lambda_{2}}\right)^{2}=\frac{\left(\frac{A a}{\lambda_{1}}+\frac{b B}{\lambda_{2}} \eta^{2}\right)^{2}}{\eta^{2}}$

We consider the difference

$$
\begin{aligned}
& A B\left(\frac{a}{\lambda_{1}}+\frac{b}{\lambda_{2}} \eta^{2}\right)^{2}-\left(\frac{A a}{\lambda_{1}}+\frac{b B}{\lambda_{2}} \eta^{2}\right)^{2} \\
= & (B-A) \cdot\left[A\left(\frac{a}{\lambda_{1}}\right)^{2}-B\left(\frac{b}{\lambda_{2}} \eta^{2}\right)^{2}\right] .
\end{aligned}
$$

From (41), we have

$$
\frac{a}{\lambda_{1}}=\frac{\delta_{1}}{\lambda_{1}} \frac{\varphi_{1}}{\varphi_{2}}, \quad \frac{b}{\lambda_{2}} \eta^{2}=\frac{\gamma_{2}}{\lambda_{1}} \frac{\lambda_{2}}{\lambda_{1}} \frac{\varphi_{1}}{\varphi_{2}} .
$$

In the case where $f\left(H^{*}, V^{*}\right)=\frac{k V^{* 2}}{H^{*}}-g C<0$, from the expression of $\delta_{1}$ in (33), we have $\delta_{1}>0$, thus $a>0$. Moreover, we consider the following two cases for $\gamma_{2}$

(a) For any fixed $x \in[0, L]$, if $\gamma_{2}(x)>0$, then we have $b>0$. From (33) and (34), we have

$$
\gamma_{2}(x)<\delta_{1}(x)
$$

Noticing $\lambda_{2}<\lambda_{1}$, we conclude that

$$
\frac{b}{\lambda_{2}} \eta^{2}=\frac{\gamma_{2}}{\lambda_{1}} \frac{\lambda_{2}}{\lambda_{1}} \frac{\varphi_{1}}{\varphi_{2}}<\frac{\delta_{1}}{\lambda_{1}} \frac{\varphi_{1}}{\varphi_{2}}=\frac{a}{\lambda_{1}} .
$$

(b) For any fixed $x \in[0, L]$, if $\gamma_{2}(x)<0$, i.e., $b<0$. From the fact that

$$
\frac{a}{\lambda_{1}}+\frac{b}{\lambda_{2}} \eta^{2}>0
$$


we get

$$
\left|\frac{b}{\lambda_{2}} \eta^{2}\right|<\frac{a}{\lambda_{1}}
$$

Above all, we get from (74) and (75) that

$$
\left|\frac{b}{\lambda_{2}} \eta^{2}\right|<\frac{a}{\lambda_{1}}, \quad \forall x \in[0, L] .
$$

Hence, we can choose $A$ and $B$ such that

$$
\max _{x \in[0, L]}\left(\left(\frac{b}{\lambda_{2}} \eta^{2}\right)^{2}\left(\frac{\lambda_{1}}{a}\right)^{2}\right) \cdot B<A<B .
$$

From (70) to (73) and noticing (77), we obtain

$$
-\left(\lambda_{1} f_{1}\right)_{x}\left(\lambda_{2} f_{2}\right)_{x}>\left(f_{1} a+f_{2} b\right)^{2}, \quad \forall x \in[0, L] .
$$

Moreover, noticing the definition of $\eta$ in (27), we can choose $A$ and $B$ such that $A / B$ is sufficiently close to 1 to guarantee the negativeness of the boundary term (60).

3.2 Exponential stability of the steady-state of the nonlinear system in $\mathrm{H}^{2}$-norm

We will now prove our main result, Theorem 3. Firstly, we recall the following theorem which gives sufficient conditions for the exponential stability of the steadystate of the nonlinear system (1), (2) and (7).

Theorem 7 The steady-state $\left(H^{*}, V^{*}\right)^{T}$ of the system (1), (2) and (7) is exponentially stable for the $H^{2}$-norm if

- There exists two functions $f_{1}, f_{2} \in C^{1}([0, L] ;(0,+\infty))$ such that

$$
-\left(\lambda_{1} f_{1}\right)_{x}>0, \quad\left(\lambda_{2} f_{2}\right)_{x}>0
$$

and

$$
-\left(\lambda_{1} f_{1}\right)_{x}\left(\lambda_{2} f_{2}\right)_{x}>\left(\frac{a(x)}{\lambda_{1}(x)} f_{1}(x)+\frac{b(x)}{\lambda_{2}(x)} f_{2}(x)\right)^{2}
$$

for any $x \in[0, L]$, where $a$ and $b$ are given by (41).

- The following inequalities are satisfied:

$$
\begin{aligned}
& \left(\frac{b_{0} H^{*}(0)+\sqrt{g H^{*}(0)}}{b_{0} H^{*}(0)-\sqrt{g H^{*}(0)}}\right)^{2}<\frac{\lambda_{2}(0) f_{2}(0)}{\lambda_{1}(0) f_{1}(0)} \varphi^{-2}(0), \\
& \left(\frac{b_{1} H^{*}(L)-\sqrt{g H^{*}(L)}}{b_{1} H^{*}(L)+\sqrt{g H^{*}(L)}}\right)^{2}<\frac{\lambda_{1}(L) f_{1}(L)}{\lambda_{2}(L) f_{2}(L)} \varphi^{2}(L),
\end{aligned}
$$

where $b_{0}, b_{1}$ and $\varphi$ are given by (21) and (38) respectively.
Remark 8 This theorem comes directly from [3, Theorem 6.6 and 6.10]. Note that finding such $f_{1}$ and $f_{2}$ corresponds to finding a quadratic Lyapunov function $V$ for the $H^{2}$-norm of the perturbations (15) and (16) such that:

$\frac{1}{\beta}\left\|(h, v)^{T}\right\|_{H^{2}} \leq V \leq \beta\left\|(h, v)^{T}\right\|_{H^{2}} \quad$ and $\quad \frac{d V}{d t} \leq-\alpha V$

for some $\alpha>0$ and $\beta>0$. In particular, such Lyapunov function has some robustness with respect to small perturbations of the system dynamics. More details about the construction of such Lyapunov function as well as the proof of this theorem can be found in the Appendix.

Using Theorem 7, we shall finally prove Theorem 3 that is now straightforward.

PROOF. [Proof of Theorem 3] Note that the condition (78) and (79) are exactly the same with conditions (50) and (51). Therefore from the proof of Proposition 4 for all $\varepsilon \in\left(0, \varepsilon_{0}\right]$, there exist $f_{1}=f_{1, \varepsilon}$ and $f_{2}=f_{2, \varepsilon}$ defined by (57), continuous with respect to $\varepsilon$, such that (78) and (79) are verified and

$$
\frac{f_{1,0} \lambda_{1}}{f_{2,0} \lambda_{2}} \varphi^{2}=\left(\frac{\lambda_{1}}{\lambda_{2}}\right)^{2}
$$

where $\varphi$ is given by (38). Under hypothesis (14) of Theorem 3 , we have (44), which together with (82) gives (61). Recall that by the continuity of $f_{1, \varepsilon}$ and $f_{2, \varepsilon}$ with respect to $\varepsilon,(63)$ holds for any $\varepsilon \in\left(0, \varepsilon_{1}\right]$. Combining (61), (63) and noticing (43), we obtain that

$$
\begin{aligned}
& \left(\frac{b_{0} H^{*}(0)+g H^{*}(0)}{b_{0} H^{*}(0)-g H^{*}(0)}\right)^{2}<\frac{\lambda_{2}(0) f_{2, \varepsilon}(0)}{\lambda_{1}(0) f_{1, \varepsilon}(0)} \varphi^{-2}(0), \\
& \left(\frac{b_{1} H^{*}(L)-g H^{*}(L)}{b_{1} H^{*}(L)+g H^{*}(L)}\right)^{2}<\frac{\lambda_{1}(L) f_{1, \varepsilon}(L)}{\lambda_{2}(L) f_{2, \varepsilon}(L)} \varphi^{2}(L) .
\end{aligned}
$$

Thus, we get from Theorem 7 that the steady-state $\left(H^{*}, V^{*}\right)^{T}$ of the system (1), (2) and (7) is exponentially stable for the $\mathrm{H}^{2}$-norm. This ends the proof of Theorem 3.

Remark 9 We emphasize that the exponential stability in $H^{p}$-norm holds in fact for any $p \in \mathbb{N} \backslash\{0,1\}$ under the same condition (14) given in Theorem 3 when the map $\mathcal{B}$ is of class $C^{p}$ and the definition of the exponential stability involves an appropriate extension of the compatibility conditions of order $p-1$ (see [3, Page.153] for the definition). This is a consequence of [3, Theorem 6.10]. Roughly speaking, this can be obtained by considering the augmented systems and then using the same method as in the proof of Theorem 7. 


\section{Conclusion}

In this paper we addressed the problem of the exponential stability of the Saint-Venant equations with arbitrary friction and space-varying slope. An explicit boundary condition was given which guarantees the exponential stability of the nonlinear system in $\mathrm{H}^{2}$-norm. To that end, we first studied a corresponding linearized system and proved the exponential stability result in $L^{2}$-norm by constructing a quadratic Lyapunov function. Then by expanding the Lyapunov function, we obtained the exponential stability of the nonlinear system in $H^{2}$-norm by requiring proper conditions on the boundaries. These boundary conditions are related to physical devices located at the ends of the channel where the controls acting as feedback are implemented.

\section{A Appendix}

\section{A.1 Proof of Lemma 5}

PROOF. From (3), (5), (32) to (38) and (41), we get that

$$
\begin{aligned}
& \left(\frac{\lambda_{2}}{\lambda_{1}} \varphi\right)^{\prime}=\frac{\lambda_{2}^{\prime} \lambda_{1}-\lambda_{1}^{\prime} \lambda_{2}}{\lambda_{1}^{2}} \varphi+\frac{\lambda_{2}}{\lambda_{1}}\left(\frac{\gamma_{1}}{\lambda_{1}}+\frac{\delta_{2}}{\lambda_{2}}\right) \varphi \\
& =\left(\frac{3 \sqrt{g H^{*}} V^{*}\left(g C-\frac{k V^{* 2}}{H^{*}}\right)}{\lambda_{1}^{2}\left(g H^{*}-V^{* 2}\right)}+\frac{\lambda_{2} \gamma_{1}+\delta_{2} \lambda_{1}}{\lambda_{1}^{2}}\right) \varphi \\
& =\left(\frac{3 \sqrt{g H^{*}} V^{*}\left(g C-\frac{k V^{* 2}}{H^{*}}\right)}{\lambda_{1}^{2}\left(g H^{*}-V^{* 2}\right)}\right. \\
& +\frac{1}{\lambda_{1}^{2}}\left[-\frac{3}{4}\left(g C-\frac{k V^{* 2}}{H^{*}}\right)\left[\frac{\sqrt{g H^{*}}-V}{\sqrt{g H^{*}}+V}-\frac{\sqrt{g H^{*}}+V}{\sqrt{g H^{*}}-V}\right]\right. \\
& \left.\left.+\frac{k V^{* 2}}{H^{*}}\left(\frac{2 \sqrt{g H^{*}}}{V^{*}}+\frac{V^{*}}{\sqrt{g H^{*}}}\right)\right]\right) \varphi \\
& =\frac{\left(\frac{3 \sqrt{g H^{*}} V^{*}\left(g C-\frac{k V^{* 2}}{H^{*}}\right)}{\lambda_{1}^{2}\left(g H^{*}-V^{* 2}\right)}-\frac{1}{\lambda_{1}^{2}}\left[\frac{3\left(g C-\frac{k V^{* 2}}{H^{*}}\right) \sqrt{g H^{*}} V^{*}}{g H^{*}-V^{* 2}}\right.\right.}{} \\
& \left.\left.=\frac{k V^{* 2}}{H^{*}}\left(\frac{2 \sqrt{g H^{*}}}{V^{*}}+\frac{V^{*}}{\sqrt{g H^{*}}}\right)\right]\right) \varphi \\
& V^{* 2}\left(\frac{2 \sqrt{g H^{*}}}{V^{*}}+\frac{V^{*}}{\sqrt{g H^{*}}}\right) \frac{\varphi}{\lambda_{1}^{2}} .
\end{aligned}
$$

Besides, we have

$$
\begin{aligned}
\frac{a}{\lambda_{1}}+\frac{b}{\lambda_{2}} \eta^{2} & =\left(\frac{\delta_{1} \lambda_{1}+\gamma_{2} \lambda_{2}}{\lambda_{1}^{2}}\right) \varphi \\
& =\frac{k V^{* 2}}{H^{*}}\left(\frac{2 \sqrt{g H^{*}}}{V^{*}}+\frac{V^{*}}{\sqrt{g H^{*}}}\right) \frac{\varphi}{\lambda_{1}^{2}}>0 .
\end{aligned}
$$

Therefore

$$
\eta^{\prime}=\frac{a}{\lambda_{1}}+\frac{b}{\lambda_{2}} \eta^{2}=\left|\frac{a}{\lambda_{1}}+\frac{b}{\lambda_{2}} \eta^{2}\right| .
$$

This ends the proof of Lemma 5 .

\section{A.2 Proof of Theorem 7}

PROOF. This theorem is a particular case of [3, Theorem 6.10]. One just need to check that the system (1), (2) and (8) with boundary conditions (7) satisfying the dissipative conditions (14) can be written in the form of $[3,(6.54)-(6.57)]$. Note that this also implies the wellposedness of the system as well. Indeed, we perform the change of variable

$$
\left(\begin{array}{c}
z_{1} \\
z_{2}
\end{array}\right)=\left(\begin{array}{cc}
\varphi_{1} \sqrt{\frac{g}{H^{*}}} & \varphi_{1} \\
-\varphi_{2} \sqrt{\frac{g}{H^{*}}} & \varphi_{2}
\end{array}\right)\left(\begin{array}{l}
h \\
v
\end{array}\right)
$$

where $h$ and $v$ are the perturbations given by (15) and (16) and $\varphi_{1}$ and $\varphi_{2}$ are given by (36) and (37) respectively. If we denote by $\mathbf{z}=\left(z_{1}, z_{2}\right)^{T}$, the nonlinear system (1), (2) and (7) is equivalent to:

$$
\begin{gathered}
\mathbf{z}_{t}+A(\mathbf{z}, x) \mathbf{z}_{x}+B(\mathbf{z}, x)=0 \\
\left(\begin{array}{c}
z_{1}(t, 0) \\
z_{2}(t, L)
\end{array}\right)=G\left(\begin{array}{c}
z_{1}(t, L) \\
z_{2}(t, 0)
\end{array}\right),
\end{gathered}
$$

where

$$
\begin{aligned}
& A(\mathbf{0}, x)=\left(\begin{array}{cc}
\lambda_{1} & 0 \\
0 & -\lambda_{2}
\end{array}\right), B(\mathbf{0}, x)=0 \\
& \text { and } \frac{\partial B}{\partial \mathbf{z}}(\mathbf{0}, x)=\left(\begin{array}{cc}
0 & a(x) \\
b(x) & 0
\end{array}\right)
\end{aligned}
$$

and

$$
G(\mathbf{0})=\mathbf{0}, \quad G^{\prime}(\mathbf{0})=\left(\begin{array}{cc}
0 & k_{0} \varphi(0) \\
k_{1} \varphi^{-1}(L) & 0
\end{array}\right)
$$


with $k_{0}$ and $k_{1}$ defined in (43). Note that the boundary condition (A.6) obtained from (7) is true at least locally, thus is in the form used in [3, Theorem 6.10]. To be more precise, noticing $\varphi_{1}(0)=1$, we get from (A.4) that

$$
\begin{aligned}
z_{1}(t, 0) & =v(t, 0)+\sqrt{\frac{g}{H^{*}(0)}} h(t, 0) \\
& =V(t, 0)-V^{*}(0)+\sqrt{\frac{g}{H^{*}(0)}} h(t, 0) \\
& =\mathcal{B}(H(t, 0))-V^{*}(0)+\sqrt{\frac{g}{H^{*}(0)}} h(t, 0) \\
& =\mathcal{B}\left(h(t, 0)+H^{*}(0)\right)-V^{*}(0)+\sqrt{\frac{g}{H^{*}(0)}} h(t, 0) \\
& :=l_{1}(h(t, 0)) .
\end{aligned}
$$

Similarly note that $\varphi_{2}(0)=1$, we obtain

$$
\begin{aligned}
z_{2}(t, 0) & =v(t, 0)-\sqrt{\frac{g}{H^{*}(0)}} h(t, 0) \\
& =\mathcal{B}\left(h(t, 0)+H^{*}(0)\right)-V^{*}(0)-\sqrt{\frac{g}{H^{*}(0)}} h(t, 0) \\
& :=l_{2}(h(t, 0)) .
\end{aligned}
$$

From (21) and (A.9), we have

$$
l_{2}^{\prime}(0)=\mathcal{B}^{\prime}\left(H^{*}(0)\right)-\sqrt{\frac{g}{H^{*}(0)}}=b_{0}-\sqrt{\frac{g}{H^{*}(0)}},
$$

which together with the definition of $b_{0}$ in (23) gives that

$$
l_{2}^{\prime}(0)<0
$$

Thanks to the implicit function theorem, we get from (A.8), (A.9) and (A.11) that in a neighborhood of 0

$$
z_{1}(t, 0)=m_{1}\left(z_{2}(t, 0)\right)
$$

Similarly, we can obtain in a neighborhood of 0 that

$$
z_{2}(t, L)=m_{2}\left(z_{1}(t, L)\right)
$$

Note that (A.12) and (A.13) are indeed in the form of (A.6). Then [3, Theorem 6.6 and 6.10] can be directly applied to this particular case and gives the sufficient conditions (78), (79) and (80). We remark here that the essential element of the proof for [3, Theorem 6.6 and $6.10]$ is that finding such $f_{1}$ and $f_{2}$ corresponds to finding a quadratic Lyapunov function for the $H^{2}$-norm of the form:

$$
\begin{aligned}
V= & \int_{0}^{L}\left(f_{1}(x) z_{1}^{2}(t, x)+f_{2}(x) z_{2}^{2}(t, x)\right) d x \\
& +\int_{0}^{L}\left(f_{1}(x) z_{1 t}^{2}(t, x)+f_{2}(x) z_{2 t}^{2}(t, x)\right) d x \\
& +\int_{0}^{L}\left(f_{1}(x) z_{1 t t}^{2}(t, x)+f_{2}(x) z_{2 t t}^{2}(t, x)\right) d x .
\end{aligned}
$$

One can look at in particular Lemma 6.8 and (6.19) to (6.22) in [3]. This completes the statement of Remark 8.

\section{Acknowledgements}

The authors would like to thank Jean-Michel Coron, Tatsien Li and Sébastien Boyaval for their constant support. The authors would like also to thank Georges Bastin, Shengquan Xiang and Christophe Zhang for many fruitful disscussions. Finally, the authors would like to thank National Natural Science Foundation of China (No. 11771336), ETH-ITS, ETH-FIM, ANR project Finite4SoS (No.ANR 15-CE23-0007) and the French Corps des IPEF for their financial support.

\section{References}

[1] Jean Auriol and Florent Di Meglio. Minimum time control of heterodirectional linear coupled hyperbolic PDEs. Automatica J. IFAC, 71:300-307, 2016.

[2] Georges Bastin and Jean-Michel Coron. On boundary feedback stabilization of non-uniform linear $2 \times 2$ hyperbolic systems over a bounded interval. Systems 8 Control Letters, 60(11):900-906, 2011.

[3] Georges Bastin and Jean-Michel Coron. Stability and Boundary Stabilisation of 1-D Hyperbolic Systems. Number 88in Progress in Nonlinear Differential Equations and Their Applications. Springer International, 2016.

[4] Georges Bastin and Jean-Michel Coron. A quadratic Lyapunov function for hyperbolic density-velocity systems with nonuniform steady states. Systems \& Control Letters, 104:66-71, 2017.

[5] Georges Bastin, Jean-Michel Coron, and Brigitte d'Andréa Novel. On lyapunov stability of linearised saint-venant equations for a sloping channel. Networks and Heterogeneous Media, 4(2):177-187, 2009.

[6] M. Hanif Chaudhry. Open-Channel Flow. Springer Publishing Company, Incorporated, 2nd edition, 2007.

[7] Jean-Michel Coron. Control and nonlinearity, volume 136 of Mathematical Surveys and Monographs. American Mathematical Society, Providence, RI, 2007.

[8] Jean-Michel Coron and Georges Bastin. Dissipative boundary conditions for one-dimensional quasi-linear hyperbolic systems: Lyapunov stability for the $C^{1}$-norm. SIAM J. Control Optim., 53(3):1464-1483, 2015. 
[9] Jean-Michel Coron, Georges Bastin, and Brigitte d'Andréa Novel. Dissipative boundary conditions for one-dimensional nonlinear hyperbolic systems. SIAM Journal on Control and Optimization, 47(3):1460-1498, 2008.

[10] Jean-Michel Coron, Brigitte d'Andréa Novel, and Georges Bastin. A lyapunov approach to control irrigation canals modeled by Saint-Venant equations. In $C D$-Rom Proceedings, Paper F1008-5, ECC99, Karlsruhe, Germany, pages 31783183, 1999.

[11] Jean-Michel Coron, Brigitte d'Andréa Novel, and Georges Bastin. A strict Lyapunov function for boundary control of hyperbolic systems of conservation laws. IEEE Transactions on Automatic Control, 52(1):2-11, 2007.

[12] Jean-Michel Coron, Long Hu, and Guillaume Olive. Finitetime boundary stabilization of general linear hyperbolic balance laws via Fredholm backstepping transformation. Automatica J. IFAC, 84:95-100, 2017.

[13] Jean-Michel Coron and Hoai-Minh Nguyen. Dissipative boundary conditions for nonlinear 1-d hyperbolic systems: sharp conditions through an approach via time-delay systems. SIAM Journal on Mathematical Analysis, 47(3):2220-2240, 2015.

[14] Jean-Michel Coron, Rafael Vazquez, Miroslav Krstic, and Georges Bastin. Local exponential $H^{2}$ stabilization of a $2 \times 2$ quasilinear hyperbolic system using backstepping. SIAM J. Control Optim., 51(3):2005-2035, 2013.

[15] Jonathan de Halleux, Christophe Prieur, Jean-Michel Coron, Brigitte d'Andréa Novel, and Georges Bastin. Boundary feedback control in networks of open channels. Automatica. A Journal of IFAC, the International Federation of Automatic Control, 39(8):1365-1376, 2003.

[16] Barré de Saint-Venant. Théorie du mouvement non permanent des eaux, avec application aux crues des rivières et à l'introduction des marées dans leur lit. Comptes Rendus de l'Académie des Sciences, 53:147-154, 1871.

[17] Florent Di Meglio, Miroslav Krstic, and Rafael Vazquez. A backstepping boundary observer for a class of linear first-order hyperbolic systems. In 2013 European Control Conference (ECC), pages 1597-1602, July 2013.

[18] Florent Di Meglio, Rafael Vazquez, and Miroslav Krstic. Stabilization of a system of $n+1$ coupled first-order hyperbolic linear PDEs with a single boundary input. IEEE Trans. Automat. Control, 58(12):3097-3111, 2013.

[19] Ababacar Diagne, Mamadou Diagne, Shuxia Tang, and Miroslav Krstic. Backstepping stabilization of the linearized saint-venant-exner model: Part ii- output feedback. In 2015 54th IEEE Conference on Decision and Control (CDC), pages 1248-1253, Dec 2015.

[20] Ababacar Diagne, Mamadou Diagne, Shuxia Tang, and Miroslav Krstic. Backstepping stabilization of the linearized saint-venant-exner model. Automatica J. IFAC, 76:345-354, 2017.

[21] Ababacar Diagne, Shuxia Tang, Mamadou Diagne, and Miroslav Krstic. State feedback stabilization of the linearized bilayer saint-venant model. IFAC-PapersOnLine, 49(8):130$135,2016$.

[22] Valérie Dos Santos and Christophe Prieur. Boundary control of open channels with numerical and experimental validations. IEEE transactions on Control systems technology, 16(6):1252-1264, 2008.

[23] James. M. Greenberg and Tatsien Li. The effect of boundary damping for the quasilinear wave equation. J. Differential Equations, 52(1):66-75, 1984.
[24] Martin Gugat and Günter Leugering. Global boundary controllability of the de St. Venant equations between steady states. Annales de l'Institut Henri Poincaré. Analyse Non Linéaire, 20(1):1-11, 2003.

[25] Martin Gugat, Günter Leugering, Simona Tamasoiu, and Ke Wang. $H^{2}$-stabilization of the isothermal Euler equations: a Lyapunov function approach. Chinese Annals of Mathematics. Series B, 33(4):479-500, 2012.

[26] Long $\mathrm{Hu}$, Florent Di Meglio, Rafael Vazquez, and Miroslav Krstic. Control of homodirectional and general heterodirectional linear coupled hyperbolic PDEs. IEEE Trans. Automat. Control, 61(11):3301-3314, 2016.

[27] Vazquez-Rafael Di Meglio Florent Hu, Long and Miroslav Krstic. Boundary exponential stabilization of 1-d inhomogeneous quasilinear hyperbolic systems.

[28] Miroslav Krstic and Andrey Smyshlyaev. Backstepping boundary control for first-order hyperbolic PDEs and application to systems with actuator and sensor delays. Systems Control Lett., 57(9):750-758, 2008.

[29] Miroslav Krstic and Andrey Smyshlyaev. Boundary Control of PDEs: A Course on Backstepping Designs, volume 16 of Advances in Design and Control. Society for Industrial and Applied Mathematics (SIAM), Philadelphia, PA, 2008.

[30] Günter Leugering and E. J. P. Georg Schmidt. On the modelling and stabilization of flows in networks of open canals. SIAM Journal on Control and Optimization, 41(1):164-180, 2002.

[31] Tatsien Li. Exact controllability for quasilinear hyperbolic systems and its application to unsteady flows in a network of open canals. Mathematical Methods in the Applied Sciences, 27(9):1089-1114, 2004.

[32] Tatsien Li, Bopeng Rao, and Zhiqiang Wang. Exact boundary controllability and observability for first order quasilinear hyperbolic systems with a kind of nonlocal boundary conditions. Discrete and Continuous Dynamical Systems. Series A, 28(1):243-257, 2010.

[33] Christophe Prieur, Joseph Winkin, and Georges Bastin. Robust boundary control of systems of conservation laws. Mathematics of Control, Signals, and Systems, 20(2):173197, 2008.

[34] Tie Hu Qin. Global smooth solutions of dissipative boundary value problems for first order quasilinear hyperbolic systems. Chinese Ann. Math. Ser. B, 6(3):289-298, 1985. A Chinese summary appears in Chinese Ann. Math. Ser. A 6 (1985), no. 4,514 .

[35] Andrey Smyshlyaev and Miroslav Krstic. Closed-form boundary state feedbacks for a class of 1-D partial integrodifferential equations. IEEE Trans. Automat. Control, 49(12):2185-2202, 2004.

[36] Rafael Vazquez, Miroslav Krstic, and Jean-Michel Coron. Backstepping boundary stabilization and state estimation of a $2 \times 2$ linear hyperbolic system. 2011 50th IEEE Conference on Decision and Control and European Control Con665 ference, pages 4937-4942, 2011. 